\title{
Immunostaining Detection of Cytomegalovirus in Gastrointestinal Biopsies: Clinicopathological Correlation at a Large Academic Health System
}

\author{
Xiaoyan Liao a, Sharon L. Reed ${ }^{\mathrm{a}, \mathrm{b}}$, Grace Y. Lin ${ }^{\mathrm{a}, \mathrm{c}}$
}

\begin{abstract}
Background: Cytomegalovirus (CMV) infection can be asymptomatic in healthy individuals but may cause serious complications in immunocompromised patients. We investigated the clinicopathological correlation of CMV in gastrointestinal (GI) biopsies at our institute between January 1, 2013 and December 31, 2015.
\end{abstract}

Methods: A total of 105 non-neoplastic GI biopsies tested positive for CMV by immunohistochemistry (IHC). The IHC results were stratified as "true positive" if $>2$ cells stained, or "rare positive" if only 1 - 2 cells stained. Clinical information including comorbidities, serum CMV viral loads, and treatment was reviewed and correlated.

Results: Overall $1 \%$ of all GI biopsies were positive for CMV by immunostaining. The most frequently involved organ was colon, followed by esophagus, stomach, ileum and duodenum. When $>2$ cells were stained positive, serum CMV viral loads were positive in $52.2 \%$, negative in $17.2 \%$, and not tested in $27.6 \%$ of cases. When only $1-2$ cells stained positive, CMV viral loads were positive in $23.4 \%$, negative in $25.5 \%$, and not tested in $51.1 \%$ of cases. We further showed that clinical management of CMV differs based on both pathological findings and underlying diseases.

Conclusions: The role of CMV in GI biopsies remains controversial. We propose an algorithm of performing CMV immunostaining based on clinicopathological correlation.

Keywords: Cytomegalovirus; Gastrointestinal biopsy; Immunostaining

\section{Introduction}

Cytomegalovirus (CMV), a member of the herpesviridae fam-

\section{Manuscript accepted for publication November 11, 2016}

aDepartment of Pathology, University of California, San Diego, CA, USA bDepartment of Medicine, University of California, San Diego, CA, USA cCorresponding Author: Grace Y. Lin, Department of Pathology and Laboratory Medicine, UC San Diego Health System, 200 W. Arbor Dr., San Diego, CA 92103, USA. Email: g4lin@ucsd.edu

doi: https://doi.org/10.14740/gr725e ily, is a double-stranded DNA virus that replicates in the host's nucleus and manifests histopathologically as large intranuclear and smaller cytoplasmic inclusion bodies [1, 2]. Around $40-100 \%$ of the world's population is seropositive for CMV [3]. Primary CMV infection in immunocompetent individuals is mild and clinically asymptomatic [4], followed by a latent state, during which the virus remains in endothelial cells, macrophages or granulocyte stem cells, but viral proliferation is inhibited by cell-mediated immunity [5]. Failure of immune containment leads to reactivation with viral proliferation and severe systemic illness characterized by fever, pancytopenia, and inflammatory changes in liver, lungs, retina, and gastrointestinal (GI) system [6].

Tissue-invasive CMV infection/reactivation manifests histopathologically as inflammation and ulceration [7]. In CMV colitis, the body initially mounts an inflammatory response that results in watery diarrhea [8]. As ulcers increase in depth, erosion into blood vessels can cause profuse bloody diarrhea $[9,10]$. Over time, severe inflammation and vasculitis may lead to ischemia and transmural necrosis of the bowel, causing perforation and peritonitis [11]. It is estimated that CMV colitis occurs in $2-16 \%$ of patients who have received solid organ transplants, $3-5 \%$ of patients with HIV infection or acquired immunodeficiency syndrome (AIDS), and 4-16\% in patients with inflammatory bowel disease (IBD) $[12,13]$. The role of CMV in IBD patients is unclear. Some authors propose that CMV does not interfere with the clinical evolution of Crohn's disease (CD), and its involvement in ulcerative colitis (UC) is debated, especially in severe flare-ups [14].

Diagnosis of CMV infection/reactivation in biopsied tissues is classically based on histopathological identification of virus-infected cells (viral cytopathic effect) on hematoxylineosin (H\&E) stained slides, and/or detection of CMV intranuclear inclusions by immunohistochemistry (IHC) studies [15]. Although IHC may not be the most sensitive method for detecting $\mathrm{CMV}$, it is widely used in many academic centers and private practice. There are currently no standard criteria when IHC should be ordered. Whether CMV IHC should be performed routinely on biopsies with moderate and severe inflammation is still under debate $[16,17]$. Some pathologists have a lower threshold for ordering the test to avoid missing any CMV-positive cells, a potentially treatable disease, while others would consider routine immunostaining to be inefficient use of resources without clinical significance. Furthermore, when immunostaining yields ambiguous results with rare cells 

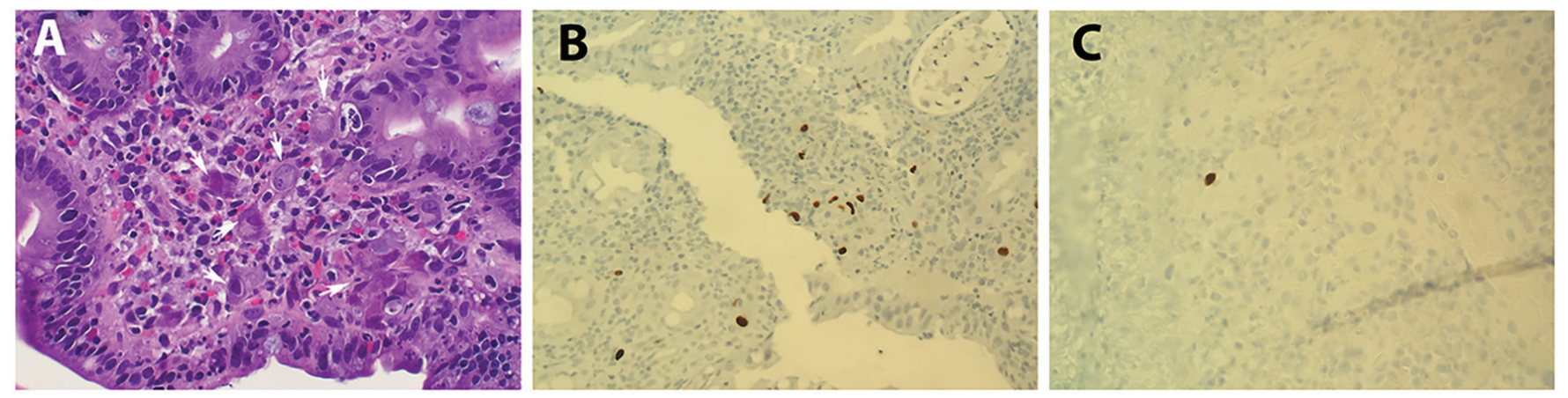

Figure 1. Histopathology of CMV. (A) H\&E staining showing CMV cytopathic effect (arrows; magnification: $\times 400)$. (B) IHC staining with CMV as positive ( $>2$ cells stained; magnification: $\times 400)$ and $(C)$ rare positive $(1-2$ cells stained; magnification: $\times 400)$.

being stained positive, the significance of rare CMV-positive cells for clinical management and outcome is unclear.

At our institute, IHC for CMV is generally ordered per clinician's request and/or when severe inflammation such as ulceration is present on the biopsy specimen, typically in immunosuppressed individuals. With the increased volume of GI biopsies especially from IBD patients, it is unclear when this test should be ordered. We investigated the clinicopathological correlation of CMV by immunostaining in GI biopsies to provide deeper insight into the role of CMV in GI pathology as well as provide a practical guideline for better management.

\section{Materials and Methods}

Institutional Review Board approval was obtained before the initiation of this study. A total of 10,013 in-house non-neoplastic GI biopsy accessioned cases (esophagus, stomach, duodenum, ileum, and colon) performed between January 1, 2013 and December 31, 2015 at our institute were identified through the electronic pathology information system (PowerPath). A total of 1,205 accessioned biopsy cases were tested for CMV infection/reactivation by IHC studies. Cases that reported CMV positivity based only on viral cytopathic effect without IHC were excluded (three cases). Cases that have concurrent diagnosis of cancer on the same specimen were also excluded (two cases). Finally, 105 cases (from 103 patients) that reported CMV positive by IHC studies were retrieved and included in this study.

Clinical information was obtained from the patients' charts through our electronic medical record system. The following parameters were recorded: patient's age, gender, relevant underlying disease or comorbidities, clinical presentation, CMV viral loads (Roche COBAS Ampliprep TaqMan CMV test), treatment (ganciclovir/valganciclovir, surgery, etc.), and follow-up biopsies. We further classified the pre-existing conditions for each CMV-positive case as follows: 1) inflammatory bowel disease, including $\mathrm{CD}$ and $\mathrm{UC}$; 2) HIV infection or AIDS; 3) solid organ or bone marrow transplant (BMT); 4) other conditions, including patients with other GI disease, cancer other than GI system status post chemotherapy and/or radiation therapy, autoimmune disease on immunosuppression, or other unspecified diarrhea.

In all cases, pathology reports and available original H\&E slides were reviewed. IHC stains were performed with antibodies against CMV (Cell Marque, 213m-25, 1:100 dilution) in the UCSD Pathology Laboratory. Pertinent positive and negative controls (Newcome supply, part \#3240B, positive: lung tissue, negative: tonsil tissue) were included for each case. In our routine sign-out of GI biopsies, while an absolute number of positive cells was usually not reported, words like "scattered, rare, ambiguous" were used when only a few cells (mostly 1 - 2 cells) are stained, and words like "positive" or "numerous" were used when a considerable number of cells are stained. To better quantify the immunodetection of CMV positivity, we re-evaluated each CMV-positive case microscopically, and defined those that only $1-2$ cells stained positive as "rare positive", and those that have more than two cells stained positive as true "positive" (Fig. 1).

Statistical analysis, including Student's $t$-test and Fisher's exact test, was performed using SPSS software.

\section{Results}

\section{Frequency of positive CMV immunostaining}

The number of GI biopsies, CMV tests performed, and percentage CMV positivity detected between January 1, 2013 and December 31, 2015 are summarized in Figure 2A and B. As shown, the annual number of non-neoplastic GI biopsy cases have steadily increased approximately 15\% from 2013 ( $\mathrm{n}=$ $3,143)$ to $2015(\mathrm{n}=3,613)$; however, the number of CMV IHC tests performed in GI biopsies more than doubled. Since the CMV infection rate in all GI biopsies did not increase $(1.06 \%$ in $2013,1.26 \%$ in 2014 , and $0.89 \%$ in 2015 ), the positive detection rate for all the IHC tests performed decreased from $12.5 \%$ in 2013 to $8.6 \%$ in 2015 .

To determine the driving force in ordering the CMV tests, we retrieved the ordering information for all 1,208 biopsy cases that were tested for CMV IHC. Among them, 152 cases were indicated by clinician to rule out "CMV infection", which accounts for 50 cases $(32.9 \%)$ that were reported as either true positive or rare positive for CMV. The remaining 1,056 cases 


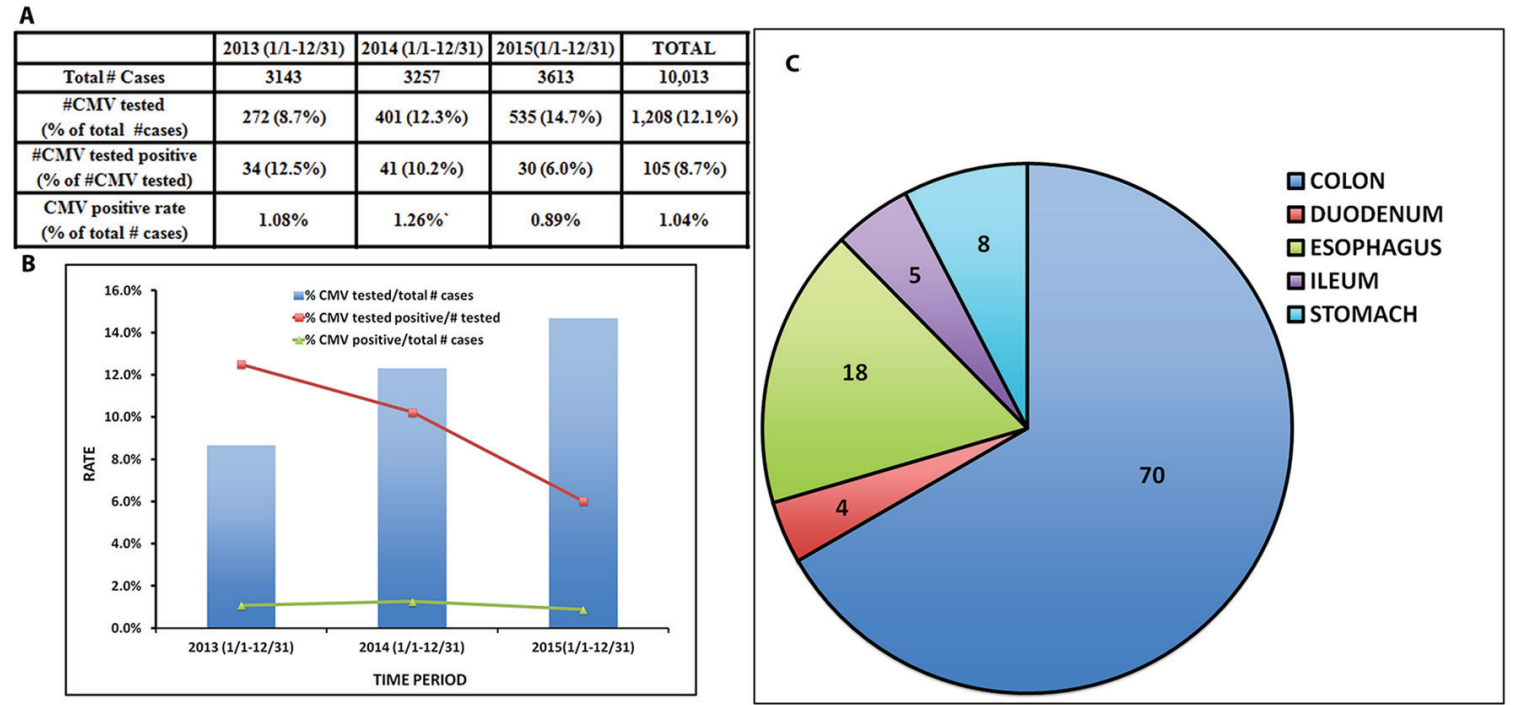

Figure 2. Summary of the total number of GI biopsies, number and percentage of CMV IHC tests performed, number and percentage of CMV positive cases between 2013 and 2015. (A) Table and (B) Bar graph. (C) Organ involvement of CMV positive cases.

tested for CMV infection by IHC were ordered by pathologists only, resulting in 55 cases $(5.2 \%)$ reported as CMV positive (Table 1).

Notably, viral cytopathic effect was described/reported in only 11 cases (10\%) among the 105 cases that were CMV-positive by immunostaining, although retrospective review of the H\&E staining in correlation with CMV staining does reveal possible viral inclusions in some cases.

\section{Demographics and outcomes of CMV-positive patients}

For patients with positive CMV on GI biopsies, the age ranged between 18 and 88 years old $($ mean $=51.8$, median $=54)$. There is slight male predominance $(\mathrm{F}: \mathrm{M}=1: 1.1)$. All patients except three survived to date. One patient (a 58-year-old female) with primary biliary sclerosis-related end-stage liver disease on steroid treatment died 1 month after CMV colitis was diagnosed. Autopsy findings revealed persistent CMV colitis and necrosis as a major source of sepsis that led to the death. The other two deceased were BMT patients that died of complications other than CMV.

Similar to other published studies [18], colon is the organ mostly frequently involved by CMV $(n=70)$, followed by esophagus $(\mathrm{n}=18)$, stomach $(\mathrm{n}=8)$, ileum $(\mathrm{n}=5)$, and duode- num $(n=4)($ Fig. 2 C).

\section{Correlation of positive IHC with CMV viremia}

Next, we reviewed the $105 \mathrm{CMV}$ immunostained slides and counted the number of CMV-positive cells on each slide. As shown in Table 2, 47 cases were classified as "rare positive" ( $\leq$ 2 cells stained) and 58 cases were classified as "positive" (>2 cells stained). We then correlated the number of CMV-positive cells with results of systemic viremia detected by CMV PCR that was performed within 1 week of the biopsies. Also shown in Table 2, cases with rare positive cells had a low percentage of positive CMV PCR (23.4\% positive, $25.5 \%$ negative, and $51.1 \%$ not tested), while cases with $>2$ positive cells have a high correlation with positive CMV PCR (55.2\% positive, $17.2 \%$ negative, and $27.6 \%$ not tested). This difference is statistically significant by Fisher's exact test $(\mathrm{P}<0.05)$, indicating that the number of cells detected by CMV IHC on GI biopsies correlated with systemic CMV viremia.

\section{Clinical management of CMV IHC positive results}

Classification of underlying conditions for the 105 CMV-posi-

Table 1. Summary of CMV IHC Tests Ordered by Clinician and Pathologists

\begin{tabular}{llllll}
\hline Time period & \#CMV IHC & $\begin{array}{l}\text { Clinician } \\
\text { requested }\end{array}$ & $\begin{array}{l}\text { CMV positive per } \\
\text { clinician's request }\end{array}$ & $\begin{array}{l}\text { Pathologist } \\
\text { requested }\end{array}$ & $\begin{array}{l}\text { CMV positive per } \\
\text { pathologist request }\end{array}$ \\
\hline 2013 (January 1 - December 31) & 272 & 43 & $20(46.5 \%)$ & 229 & $14(6.1 \%)$ \\
2014 (January 1 - December 31) & 401 & 46 & $16(37.5 \%)$ & 355 & $25(7.0 \%)$ \\
2015 (January 1 - December 31) & 535 & 63 & $14(24.1 \%)$ & 472 & $16(3.4 \%)$ \\
Total & 1,208 & 152 & $50(32.9 \%)$ & 1,056 & $55(5.2 \%)$ \\
\hline
\end{tabular}


Table 2. Correlation of the Number of Positive Cells Detected by IHC With Systemic Virology (PCR-Based Tests)

\begin{tabular}{lll}
\hline & IHC, > 2 cells labeled & IHC, $\mathbf{1}$ - 2 cells labeled \\
\hline \#CMV viral load positive* & $32(55.2 \%)$ & $11(23.4 \%)$ \\
\#CMV viral load negative & $10(17.2 \%)$ & $12(25.5 \%)$ \\
\#Virology not tested & $16(27.6 \%)$ & $24(51.1 \%)$ \\
Total & 58 & 47 \\
\hline
\end{tabular}

*VL $>137 \mathrm{IU} / \mathrm{mL}$. Fisher's exact test: $\mathrm{P}<0.05$.

tive cases and correlation of pathological findings with clinical information are summarized in Table 3 . There are a total of 40 cases with IBD (17 CD and 23 UC), 20 with HIV/AIDS, 17 with solid organ or bone marrow transplant, and 28 "others". As seen in Table 3, the majority of the 47 "rare positive" cases are from IBD patients $(\mathrm{n}=20)$, followed by "others". In contrast, patients with HIV/AIDS and those with bone marrow or solid organ transplant tend to have more CMV "positive" than "rare positive" in their biopsies.

In IBD patients, as well as patients with other conditions, if CMV immunostaining is in the category of "rare positive", the serum CMV PCR study was either negative or not tested, and as a result, the majority of these patients were not treated with anti-viral agents. In contrast, patients with HIV/AIDS or bone marrow/solid organ transplant were usually treated with antiviral medication even when only "rare positive" cells were detected (Table 3).

Interestingly, for those patients that were reported to be "rare positive" for CMV by IHC and were not clinically treated with antiviral medications, follow-up biopsies were either not performed because they were clinically not indicated, or were mostly negative for CMV if performed (data not shown).

\section{CMV in IBD patients and outcomes}

Some authors have noted that CMV mainly affects the right colon in IBD patients and does not affect the clinical course of $\mathrm{CD}$, in contrast to patients with UC [14]. Interestingly, in our study, we found that CMV was detected predominantly in the left colon for both CD and UC patients. To examine how CMV differs between these two patient groups, we selected cases with $>2$ cells positive for $\mathrm{CMV}$ on $\mathrm{IHC}(\mathrm{n}=8$ for $\mathrm{CD}$ and $\mathrm{n}=11$ for UC, Table 4). The gender distribution was similar between the two groups, while UC patients tended to be older (average 49 years) than those with CD (average 39 years). Most patients were on steroids (prednisone) around the time CMV was detected ( $\mathrm{n}=7 / 8$ in CD and $10 / 11$ in UC), and the majority of them were treated with the anti-viral agent ganciclovir ( $n=6 / 8$ in CD and 9/11 in UC) after CMV was reported. Follow-up with clinical course reveals five out of eight patients with CD and four out of 11 UC patients had very complicated clinical courses that required surgical intervention including colectomy (Table 4). Most of the surgeries occurred 0.5 - 19 months after CMV detection, while one CD patient and two UC patients underwent surgery within 1 month of CMV reactivation/infection, indicating possible association between CMV and the adverse clinical course.

\section{Discussion}

By studying the frequency and positive rate of CMV immunostaining in all non-neoplastic GI biopsy cases and correlating the pathological findings with clinical information during a 3-year period of time, we found that overall CMV infection/ reactivation rate is constant, occurring in approximately $1 \%$ of all non-neoplastic GI biopsies at our institute. The possibility of CMV is initially considered given the severity of inflammation and/or visible viral cytopathic effect on routine $\mathrm{H} \& \mathrm{E}$ staining, followed by IHC studies to confirm or rule out the presence of CMV antigen. Interestingly, lowering the threshold of ordering IHC test does not lead to increased detection of CMV positive cases, as the more IHC studies performed, the lower the positive detection rate by IHC. In our study, we further demonstrated that clinician's request to rule out CMV

Table 3. Pre-Existing Conditions Influence Clinical Management of CMV Positivity

\begin{tabular}{llll}
\hline Pre-existing disease & \# Cases & CMV by IHC & Clinical management for anti-CMV \\
\hline HIV & 20 & $\begin{array}{l}14 \text { positive } \\
6 \text { rare positive }\end{array}$ & Usually treated regardless of CMV PCR results \\
BMT or solid organ transplant & 17 & 10 positive & Usually treated regardless of CMV PCR results \\
& & 7 rare positive & \\
IBD & 40 & 20 positive & Usually treated \\
& 28 & 20 rare positive & Usually only treated if CMV PCR positive \\
Others & 28 positive & Usually treated \\
& & 14 rare positive & Usually only treated if CMV PCR positive \\
\hline
\end{tabular}


Table 4. Clinical Outcomes Bewteen CD $(n=8)$ and UC $(n=11)$ Patients With Positive CMV IHC (> 2 Cells)

\begin{tabular}{|c|c|c|c|}
\hline & $\mathrm{CD}(\mathrm{n}=8)$ & $\mathrm{UC}(\mathrm{n}=\mathbf{1 1}) *$ & Statistical test \\
\hline Age, years (range) & $39(21-77)$ & $49(21-79)$ & $\mathrm{P}>0.05$ \\
\hline Female & 3 & 5 & $\mathrm{P}>0.05$ \\
\hline On systemic steroid & 7 & 10 & $\mathrm{P}>0.05$ \\
\hline Not on steroid & 1 & 1 & \\
\hline No anti-viral treatment & 2 & 2 & \\
\hline Surgery after reported CMV & 5 & 4 & $\mathrm{P}>0.05$ \\
\hline No surgery & 3 & 7 & \\
\hline Duration from CMV to surgery if performed & $1-19$ months ( 1 within 1 month) & 0.5 - 6 months ( 2 within 1 month) & \\
\hline
\end{tabular}

*Total biopsy case number is 12 . However, there are two cases belonging to one single patient.

infection does yield a high detection rate of CMV, suggesting the importance of clinicopathological correlation.

Although immunostaining detection of CMV is relatively inexpensive with a short turn-around time, interpreting the results can sometimes be challenging. We noticed that nearly half $(45 \%)$ of the reported CMV positive biopsy specimens have actually only 1 - 2 cells stained on IHC, leading to a description of "single", "rare", "ambiguous", or comments that this finding is unlikely to be the etiology of the severe inflammation or ulceration. Indeed, whether CMV infection/reactivation represents an "innocent bystander" or "true player" in IBD disease has long been debated $[19,20]$, and data regarding CMV in this setting are quite conflicting [21-23]. Proponents of the "innocent bystander" hypothesis state that CMV virus has a propensity towards colonizing inflamed, granulated ulcer beds, and suggest that the presence of CMV represents tropism towards areas of inflammation, rather than a reflection of true disease $[21,24]$. Nonetheless, there have been several studies presenting solid evidence to support testing for CMV in cases of moderate to severe colitis, and if CMV is present, treating with ganciclovir $[15,17]$.

The dilemma of reporting "ambiguous" immunostaining results was addressed in our study by correlating the number of immunostaining positive cells on biopsies with patient's serum CMV PCR studies. While not all cases were tested for serum CMV PCR, not surprisingly, only a quarter of those cases with 1 - 2 cells stained positive have CMV viremia. In contrast, in biopsy specimens that have $>2$ cells stained, more than half of the patients were found to have systemic viremia as detected by serum PCR test. If systemic CMV viremia represents true CMV infection/re-activation, which likely it does, it is reasonable to propose that, the number of CMV-positive cells reflect the role of CMV. The fewer cells that are CMV infected, the more likely it is just an "innocent bystander"; the more cells CMV infected, the more likely it is a systemic disease contributing to the tissue pathology (severe inflammation or ulceration) seen on the biopsy specimens.

Through the pathological-clinical correlation, we also found that clinical management regarding the CMV positivity is usually based on comorbidities. Patients with HIV infection/
AIDS and those who are status post solid organ or bone marrow transplant are usually treated regardless of number of cells stained and/or serum PCR results. This is because CMV in patients with HIV/AIDS or solid organ or bone marrow transplants can cause significant disease in these immunodeficient patients [25-27]. For patients with IBD and other conditions at our institution, treatment for CMV is usually not given if only 1 - 2 cells stained, unless there is systemic viremia. In addition, most of the IBD patients with $>2$ IHC positive cells and subsequent positive CMV viral loads were on systemic steroids (prednisone) at the time of detection, suggesting that the increased risk for CMV in IBD patients may in part be iatrogenic, mostly ascribed to steroid therapy $[20,24]$. The proposal that CMV in IBD patients may represent transient CMV reactivation status/innocent bystander was further supported by negative CMV in follow-up biopsies without anti-viral treatment.

Our data suggest an algorithm that may help the pathologist address the CMV issues more practically, putting more emphasis on patients that are truly immunocompromised (HIV/ AIDS, solid organ or bone marrow transplant), while raising the threshold for testing for CMV and reporting of positivity in IBD patients to those patients who are on systemic steroids, especially when dealing with ambiguous results (Fig. 3).

CMV in IBD patients has long been a controversial topic. Although rare severe consequences of CMV infection have been reported [28, 29], studies have shown that CMV does not contribute to increased morbidity and mortality and that antiviral therapy will eradicate the virus without altering the course of underlying disease $[21,30,31]$. On the other hand, some authors proposed that CMV may alter the disease process of UC, but not CD [24]. Given the conflicting opinions, it is interesting to note in our study that the clinical course is similar in both patients with UC and CD during and after CMV, in terms of being on steroid therapy, treatment with anti-virals, and frequency of complications requiring surgical intervention. The conclusion may be limited by the small number of patient population in this study, and further follow-up study may be warranted.

In summary, we performed a study focusing on the clinicopathological correlation of CMV immunostaining in GI biopsy specimens. Our findings suggest that pathologic evalua- 


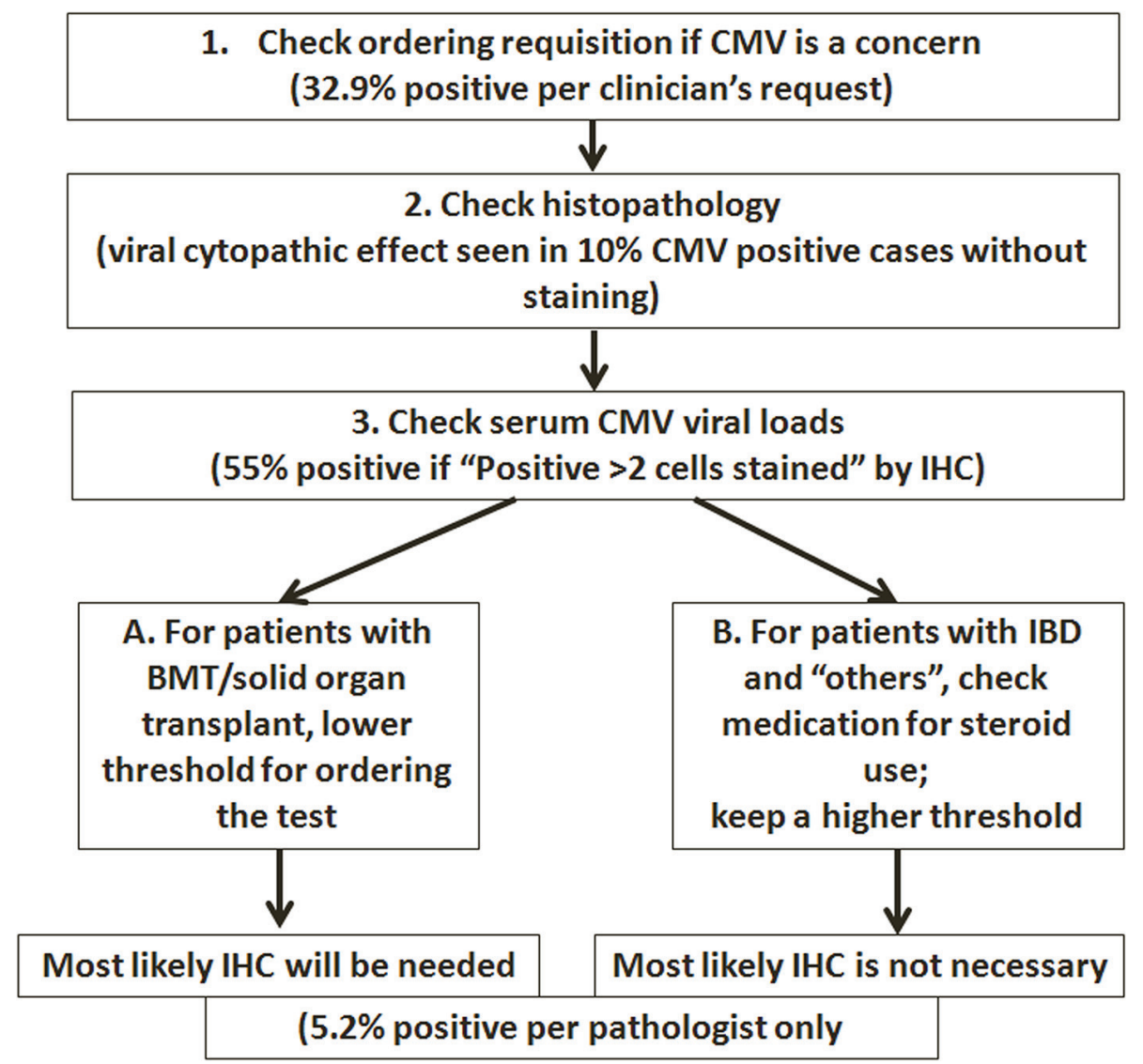

Figure 3. Algorithm proposed for performing IHC tests on GI biopsies.

tion plays an essential role in the diagnosis and management of CMV in immunocompromised patients. As an ancillary study to detect CMV, IHC studies should be ordered and interpreted in appropriate clinical context. While the threshold for ordering/ reporting CMV should be lower in patients with true immunodeficiency (such as HIV infection or status post bone marrow or solid organ transplant), knowledge of the IBD characteristics and clinical course will help to guide when the pathologist should order the test and how to interpret the results. It should be kept in mind that increasing the number of IHC tests does not lead to increased CMV detection. Finally, we propose an algorithm for performing the IHC tests in selected GI biopsies (Fig. 3), with all steps involving clinicopathological correlation.

\section{Financial Support}

The authors have no relevant financial interest in the products or companies described in this article.

\section{Disclaimer}

Presented in part at the United States \& Canadian Academy of Pathology's 105th Annual Meeting (Abstract \#1980; Poster Session V \#267), March 12 - 18, 2016 in Seattle, WA, USA.

\section{References}

1. Ryan KJ, Ray CG, Sherris JC. Sherris medical microbiology: an introduction to infectious diseases. 4th edn, McGraw-Hill. 2004;556-559.

2. Landolfo S, Gariglio M, Gribaudo G, Lembo D. The human cytomegalovirus. Pharmacol Ther. 2003;98(3):269297.

3. Lockwood MR, Liddle J, Kitsanta P. Cytomegalovirus colitis - an unusual cause for diarrhoea in an elderly woman. Age Ageing. 2006;35(2):198-200.

4. Revello MG, Zavattoni M, Sarasini A, Percivalle E, Simoncini L, Gerna G. Human cytomegalovirus in blood of immunocompetent persons during primary infection: prognostic implications for pregnancy. J Infect Dis. 1998;177(5):1170-1175.

5. La Rosa C, Diamond DJ. The immune response to human CMV. Future Virol. 2012;7(3):279-293.

6. Goodgame RW. Gastrointestinal cytomegalovirus disease. Ann Intern Med. 1993;119(9):924-935.

7. Kim JM, Kim SJ, Joh JW, Shin M, Moon JI, Jung GO, Choi GS, et al. The risk factors for cytomegalovirus syndrome and tissue-invasive cytomegalovirus disease in liver transplant recipients who have cytomegalovirus antigenemia. Transplant Proc. 2010;42(3):890-894.

8. Carter D, Olchovsky D, Pokroy R, Ezra D. Cytomegalovi- 
rus-associated colitis causing diarrhea in an immunocompetent patient. World J Gastroenterol. 2006;12(42):68986899.

9. Klauber E, Briski LE, Khatib R. Cytomegalovirus colitis in the immunocompetent host: an overview. Scand J Infect Dis. 1998;30(6):559-564.

10. Karakozis S, Gongora E, Caceres M, Brun E, Cook JW. Life-threatening cytomegalovirus colitis in the immunocompetent patient: report of a case and review of the literature. Dis Colon Rectum. 2001;44(11):1716-1720.

11. Telakis E, Tsironi E, Papatheodorou K, Nikolakis D. Debilitating chronic diarrhea caused by generalized gastrointestinal cytomegalovirus infection in an immunocompetent adult. Case Rep Gastrointest Med. 2014;2014:260120.

12. Maconi G, Colombo E, Zerbi P, Sampietro GM, Fociani $\mathrm{P}$, Bosani M, Cassinotti A, et al. Prevalence, detection rate and outcome of cytomegalovirus infection in ulcerative colitis patients requiring colonic resection. Dig Liver Dis. 2005;37(6):418-423.

13. Kim YS, Kim YH, Kim JS, Cheon JH, Ye BD, Jung SA, Park YS, et al. Cytomegalovirus infection in patients with new onset ulcerative colitis: a prospective study. Hepatogastroenterology. 2012;59(116):1098-1101.

14. Garrido E, Carrera E, Manzano R, Lopez-Sanroman A. Clinical significance of cytomegalovirus infection in patients with inflammatory bowel disease. World J Gastroenterol. 2013;19(1):17-25.

15. Lee H, Westerhoff M, Shen B, Liu X. Clinical Aspects of Idiopathic Inflammatory Bowel Disease: A Review for Pathologists. Arch Pathol Lab Med. 2016;140(5):413428.

16. Maher MM, Nassar MI. Acute cytomegalovirus infection is a risk factor in refractory and complicated inflammatory bowel disease. Dig Dis Sci. 2009;54(11):2456-2462.

17. Sager K, Alam S, Bond A, Chinnappan L, Probert CS. Review article: cytomegalovirus and inflammatory bowel disease. Aliment Pharmacol Ther. 2015;41(8):725-733.

18. Rafailidis PI, Mourtzoukou EG, Varbobitis IC, Falagas ME. Severe cytomegalovirus infection in apparently immunocompetent patients: a systematic review. Virol J. 2008;5:47.

19. Lawlor G, Moss AC. Cytomegalovirus in inflammatory bowel disease: pathogen or innocent bystander? Inflamm Bowel Dis. 2010;16(9):1620-1627.

20. Ogunneye O, Hernandez J. Acute cytomegalovirus colitis mimicking exacerbation of ulcerative colitis: precipitating or coincidental? Am J Med. 2012;125(6):e3-4.

21. Matsuoka K, Iwao Y, Mori T, Sakuraba A, Yajima T, Hisamatsu T, Okamoto S, et al. Cytomegalovirus is frequently reactivated and disappears without antiviral agents in ulcerative colitis patients. Am J Gastroenterol. 2007;102(2):331-337.

22. Domenech E, Vega R, Ojanguren I, Hernandez A, GarciaPlanella E, Bernal I, Rosinach M, et al. Cytomegalovirus infection in ulcerative colitis: a prospective, comparative study on prevalence and diagnostic strategy. Inflamm Bowel Dis. 2008;14(10):1373-1379.

23. Kuwabara A, Okamoto H, Suda T, Ajioka Y, Hatakeyama K. Clinicopathologic characteristics of clinically relevant cytomegalovirus infection in inflammatory bowel disease. J Gastroenterol. 2007;42(10):823-829.

24. Nguyen M, Bradford K, Zhang X, Shih DQ. Cytomegalovirus Reactivation in Ulcerative Colitis Patients. Ulcers. 2011;2011.

25. Dieterich DT, Rahmin M. Cytomegalovirus colitis in AIDS: presentation in 44 patients and a review of the literature. J Acquir Immune Defic Syndr. 1991;4(Suppl 1):S29-35.

26. Yoshikawa T. Significance of human herpesviruses to transplant recipients. Curr Opin Infect Dis. 2003;16(6):601-606.

27. Varani S, Landini MP. Cytomegalovirus-induced immunopathology and its clinical consequences. Herpesviridae. 2011;2(1):6.

28. Miyatani H, Yoshida Y. Retroperitoneal perforation caused by endoscopic biopsy in a patient with ulcerative colitis and cytomegalovirus infection. Endoscopy. 2007;39(Suppl 1):E246.

29. Hernandez-Camba A, Lakhwani S, Ramos L, Raya JM, Quintero E. Cytomegalovirus-associated hemophagocytic syndrome in a patient with Crohn's disease receiving azathioprine. J Gastrointestin Liver Dis. 2013;22(4):471472.

30. Eyre-Brook IA, Dundas S. Incidence and clinical significance of colonic cytomegalovirus infection in idiopathic inflammatory bowel disease requiring colectomy. Gut. 1986;27(12):1419-1425.

31. Inokuchi T, Kato J, Hiraoka S, Suzuki H, Nakarai A, Hirakawa T, Akita M, et al. Long-term follow-up of ulcerative colitis patients treated on the basis of their cytomegalovirus antigen status. World J Gastroenterol. 2014;20(2):509-517. 